Scientific Journal of Hamadan Nursing \& Midwifery Faculty - ISSN 2008-2819

\title{
Relationship Between Nursing Students' Professional Competence in Spiritual Care and Spiritual Intelligence
}

\author{
Mehrnaz Ahmadi ${ }^{1}$, Ahmad Izadi², Saeed Poormansouri ${ }^{3}$, Ladan Sedighie ${ }^{1}$, Fatemeh Estebsari ${ }^{4}$, \\ Kourosh Zarea ${ }^{*}$ \\ 1. PhD student, Student Research Committee, School of Nursing and Midwifery, Shahid Beheshti University of \\ Medical Sciences, Tehran, Iran \\ 2. Associate professor, Department of Education and Psychology, Imam Hossein University, Tehran, Iran \\ 3. MSc, Treatment Deputy, Ahvaz Jundishapur University of Medical Sciences, Ahvaz, Iran \\ 4. Assistant Professor, PhD in Health Education \& Promotion, Department of Community Health Nursing, \\ School of Nursing and Midwifery, Shahid Beheshti University of Medical Sciences (SBUMS), Tehran, Iran \\ 5. Associate professor, Nursing Care Research Center in Chronic Disease, Department of Nursing, School of \\ Nursing and Midwifery, Ahvaz Jundishapur University of Medical Sciences, Ahvaz, Iran
}

\begin{tabular}{lr}
\hline \multicolumn{2}{c}{ Article Info } \\
\hline & \\
Received: & $2017 / 07 / 12$ \\
Accepted: & $2017 / 11 / 29$
\end{tabular}

Published Online 2017/12/2

DOI: $10.30699 /$ sjhnmf.26.5.5

Original Article

Use your device to scan and read the article online

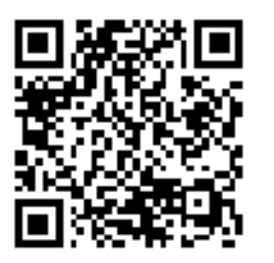

\section{Abstract}

Introduction: Spiritual care can have positive effects on physical and mental health of patients; facilitates healing process and increases patients' satisfaction. Spiritual intelligence can also be a contributing factor to nurse caring behaviors. This study aims to determine relationship between nursing students' professional competence in spiritual care and Spiritual Intelligence.

Methods: A descriptive correlation study was conducted on 140 nursing students of Ahvaz Jundishapur University of Medical Sciences in 2016. Subjects were selected using a census method. Data collection instrument consisted of demographic questions, Spiritual Intelligence and professional competence in spiritual care questionnaires. Data was analyzed using descriptive Statistics and inferential statistics including independent t-test, Kruskal-Wallis, correlation coefficient, and linear regression in SPSS19.

Results: Significant and positive correlation was found between nursing students' competence in spiritual care and Spiritual Intelligence $(P<0.001, \mathrm{r}=-0.33)$. There was only a significant difference in Spiritual Intelligence between those who had attended training courses and who didn't have these courses. Regression model indicated that for increased rates of Spiritual Intelligence, professional competence in spiritual care will rise as much as 0.54 .

Conclusion: Spiritual Intelligence can have an impact on nursing students' competencies in spiritual care. Accordingly, planning to improve the Spiritual Intelligence and nursing students' competence in spiritual care is recommended.

Keywords: Nursing Students, Professional Competence, Spiritual Care, Spiritual Intelligence

How to Cite This Article:

Ahmadi M, Izadi A, Poormansouri S, Sedighie L, Estebsari F, Zarea K. Relationship Between Nursing Students' Professional Competence in Spiritual Care and Spiritual Intelligence. Sci J Hamadan Nurs Midwifery Fac. 2018; 25 (5): 189 - 199 


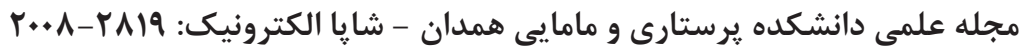

مقالل يثوهشى

\section{ارتباط بين صلاحيت حرفهاى دانشجويان رشتهُ يرستارى در حيطهُ مراقبت معنوى و هوش معنوى}

\section{مهرناز احمدى'، احمد ايزدى ‘، سعيد يور منصورى"، لادن صديقى'، فاطمه استبصارى"، كوروش زارعه"} دانشجوى دكتراى يرستارى، كميتأ يزوهشى دانشجويان، دانشكدة يرستارى و مامايى، دانشكاه علوم يزشكى و خدمات بهداشتى درمانى شهيد بهشتى، تهران، ايران

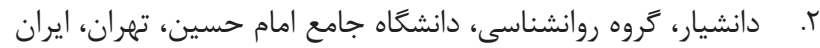

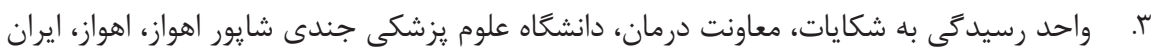

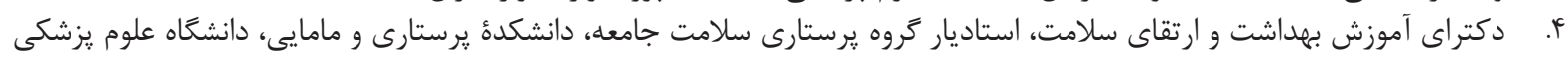

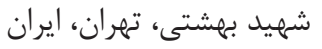

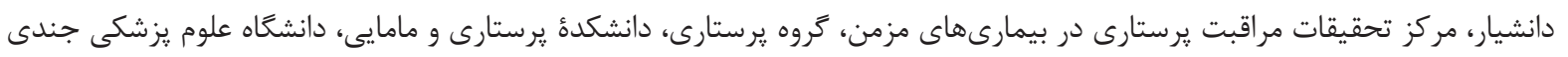
شايور اهواز، اهواز، ايران

\begin{tabular}{|c|c|}
\hline جكيده & اطلاعات مقاله \\
\hline 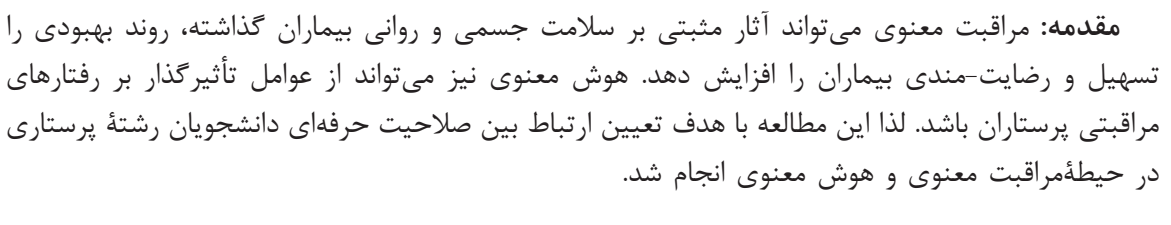 & 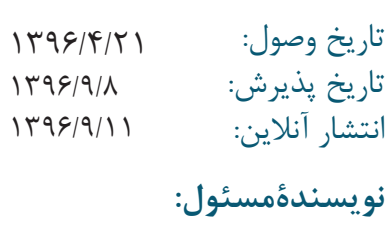 \\
\hline 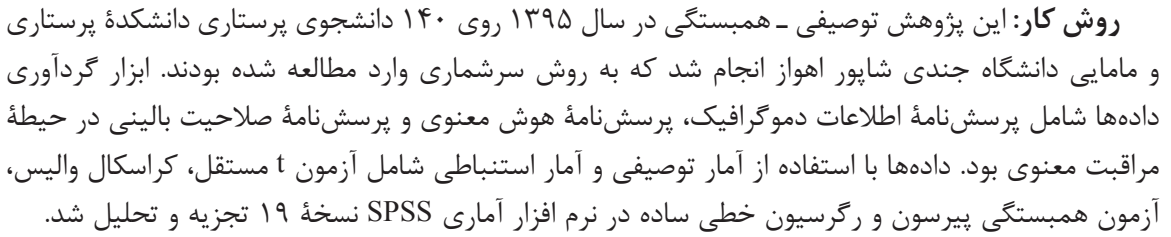 & 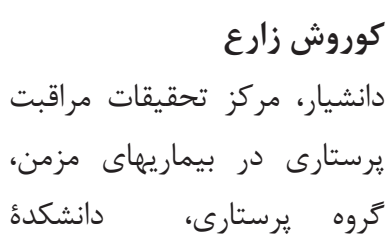 \\
\hline 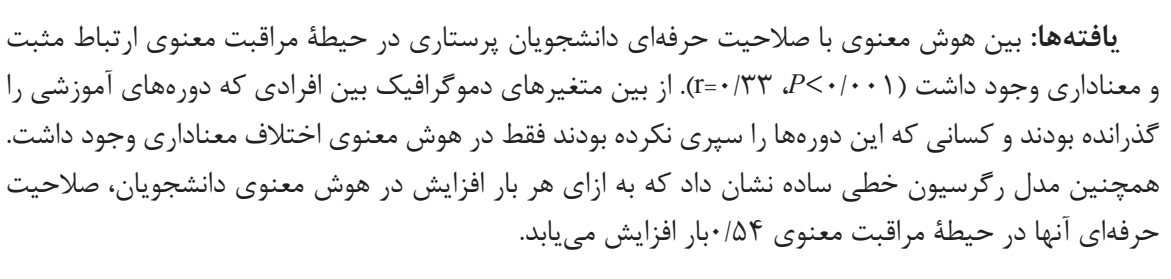 & 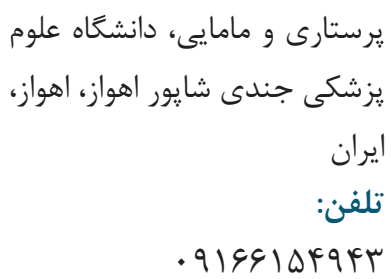 \\
\hline 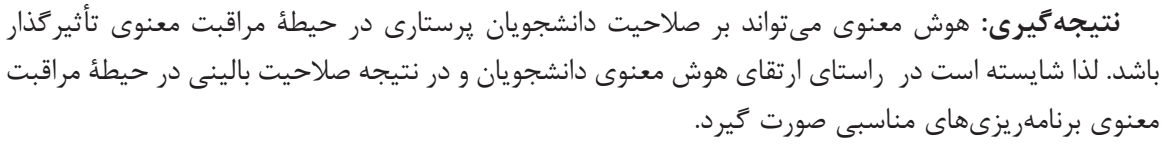 & 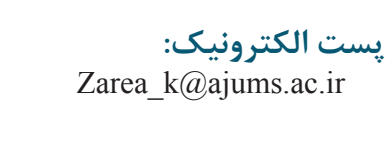 \\
\hline وازههاى كليدى: دانشجويان يرستارى، صلاحيت حرفهاى، مراقبت معنوى، هوش معنوى & \\
\hline
\end{tabular}


يرستاران در زمينهُ مراقبت معنوى باشد [ع] همانطور كه

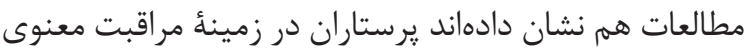

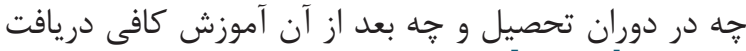

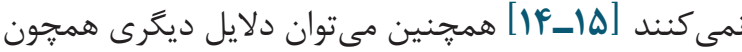

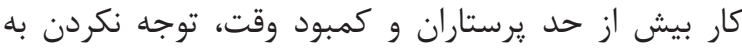

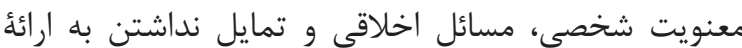

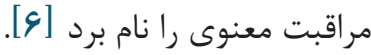

با اين حال مطالعات نشان دادهاند موارد ديخرى نيز ميتواند

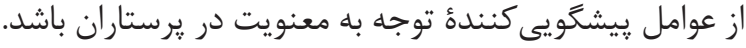

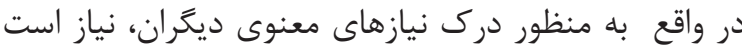

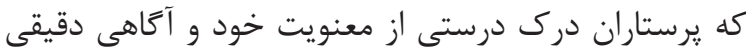

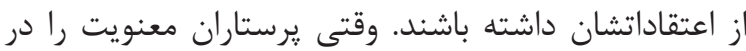

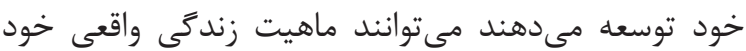

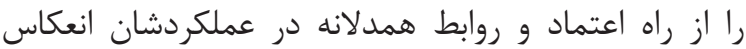

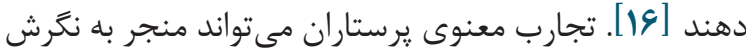

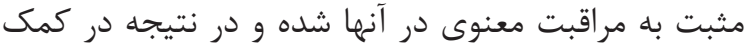

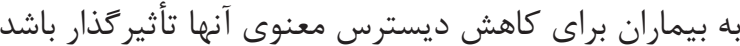

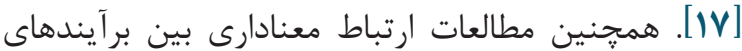

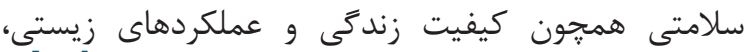

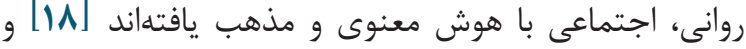

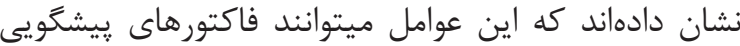

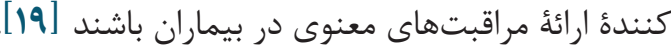

هوش معنوى به عنوان مجموعهاى از توانايىها براى

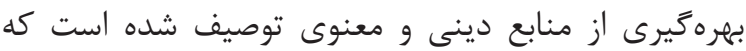

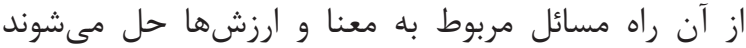

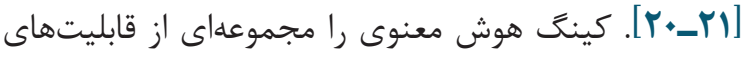

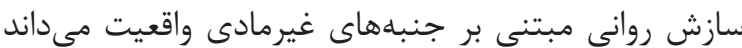

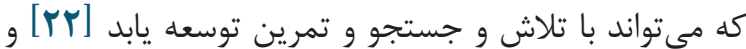

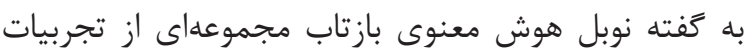

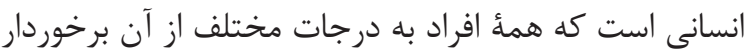

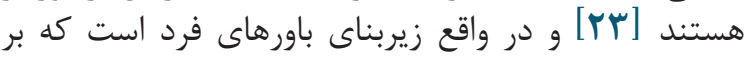

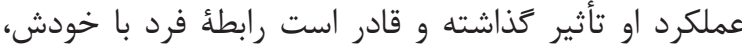

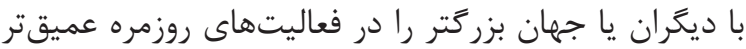

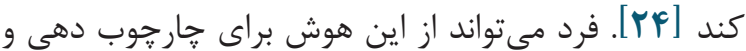

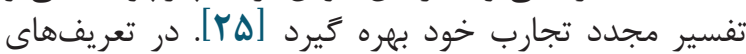

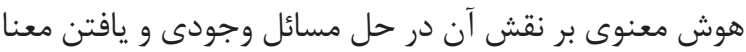

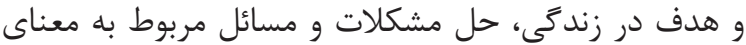

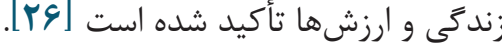

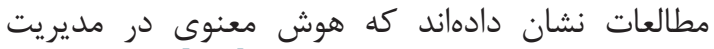

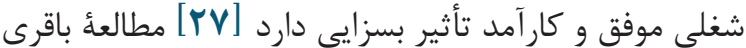

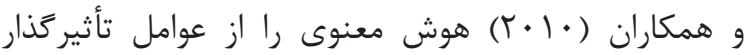

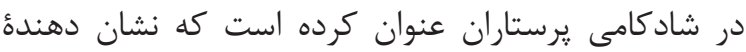

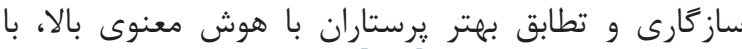

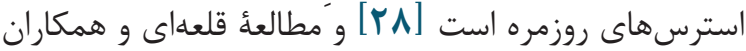

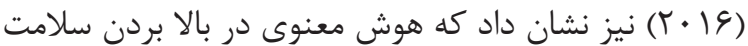

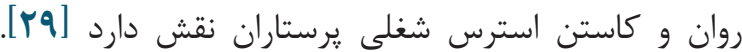

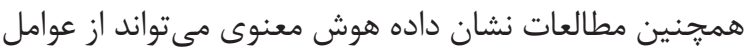

مقدمه

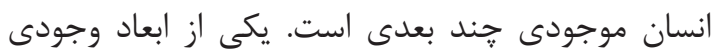

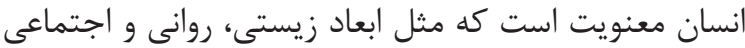

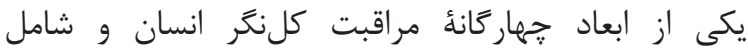

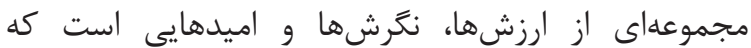

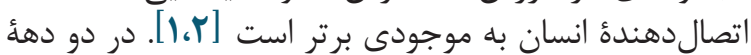

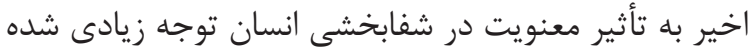

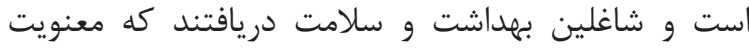

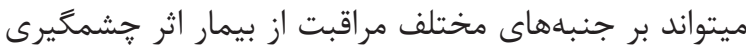

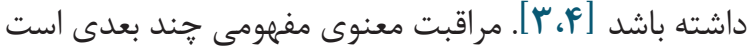

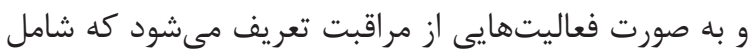

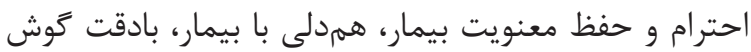

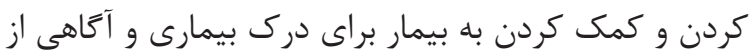

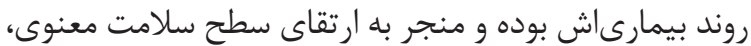

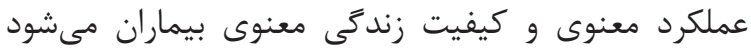

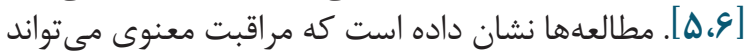

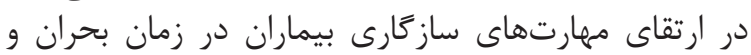

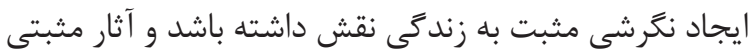

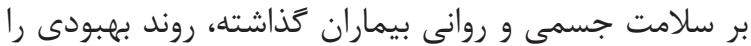

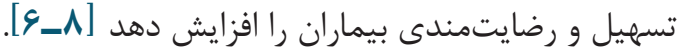

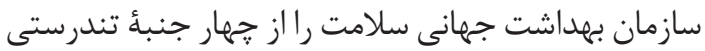

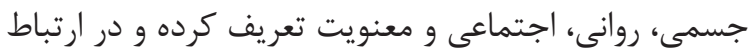

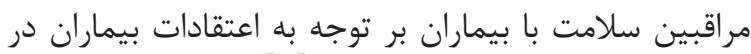

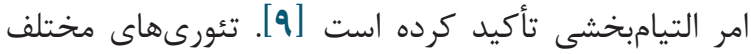

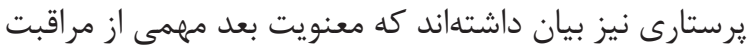

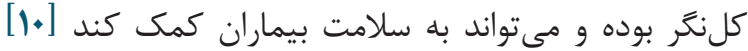

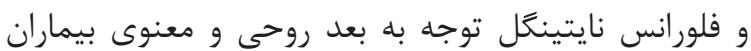

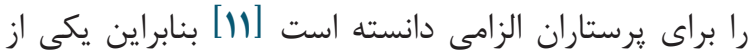

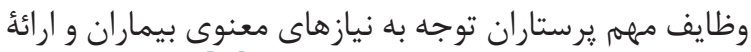

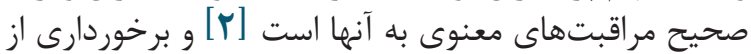

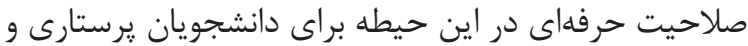

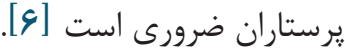
صلاحيت حرفهاى در حيطة مراقبت معنوى بهعنوان فر فرآيند

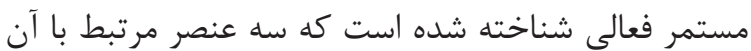

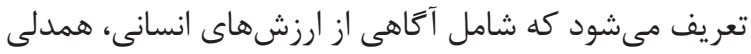

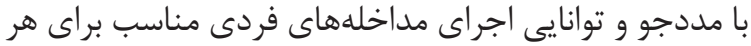

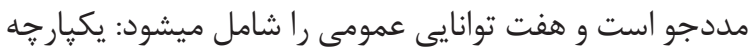

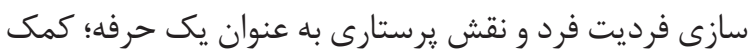

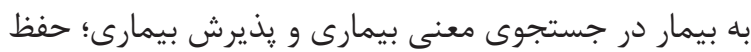

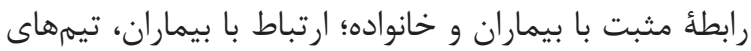

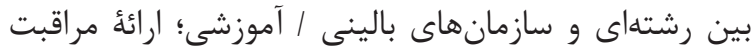

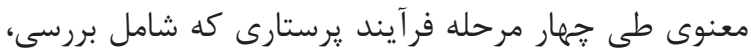

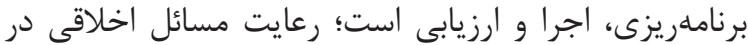

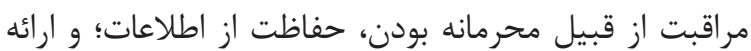

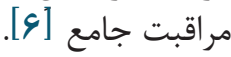

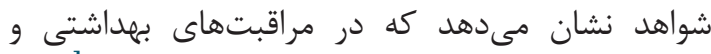

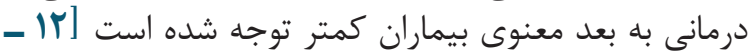

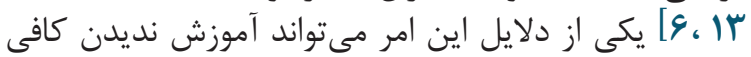




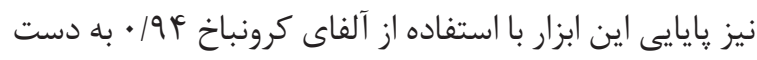

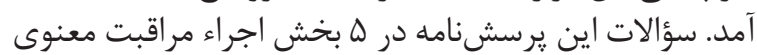

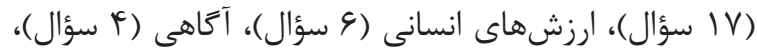

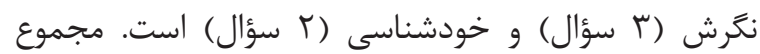

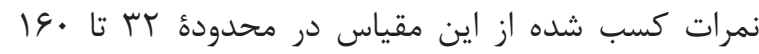

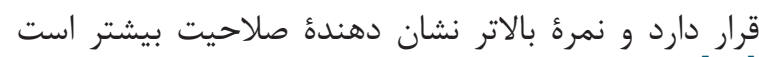

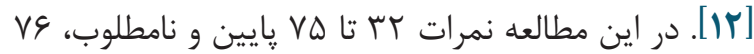

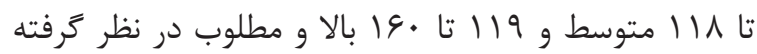

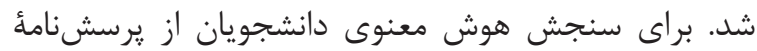

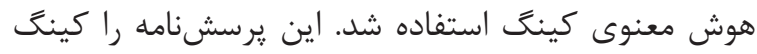

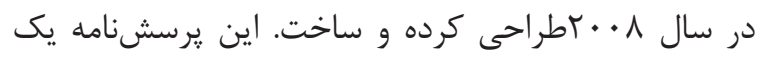

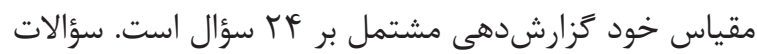

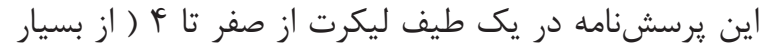

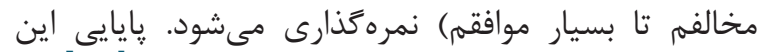

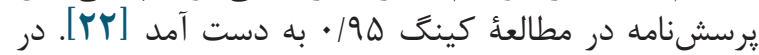

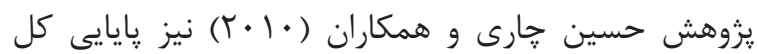

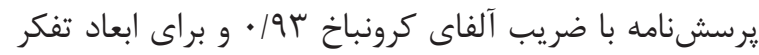

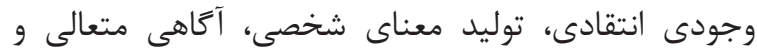

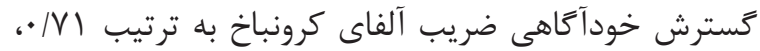

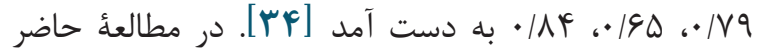

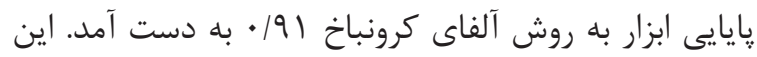

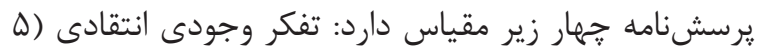

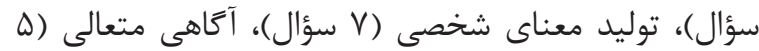

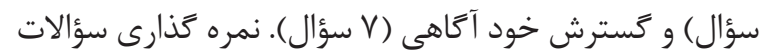

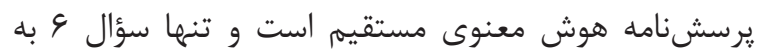

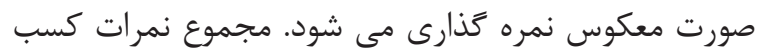

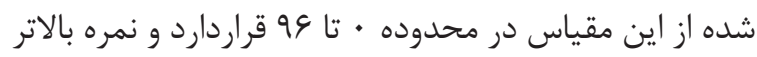

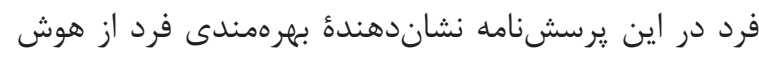

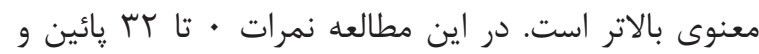

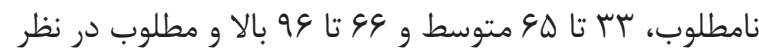

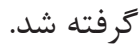

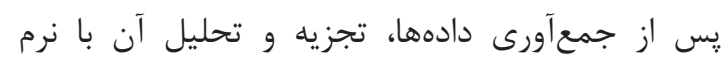

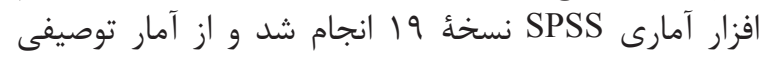

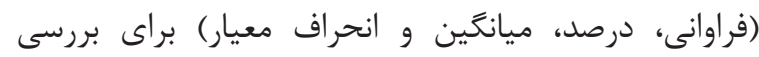

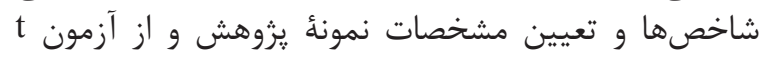

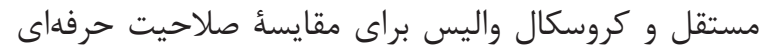

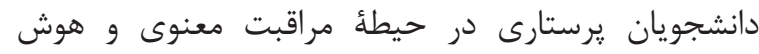

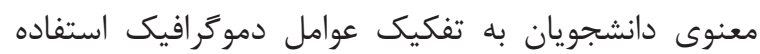

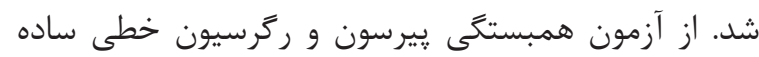

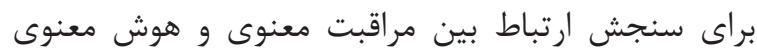

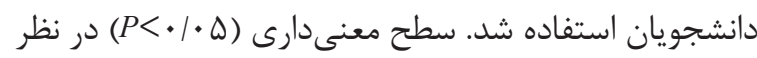
كرفته شد.

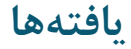

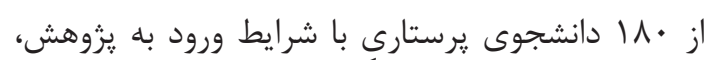

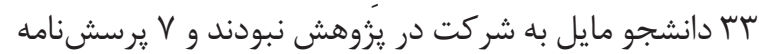

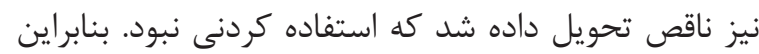

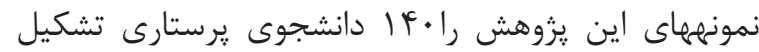

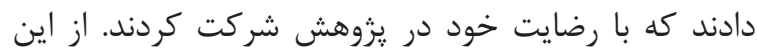

تأثير كذار بر رفتارهاى مراقبتى يرستاران باشد. مطالعأ ميرى

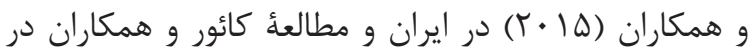

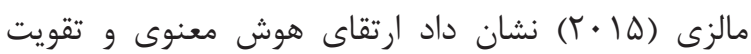

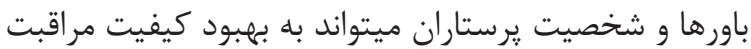

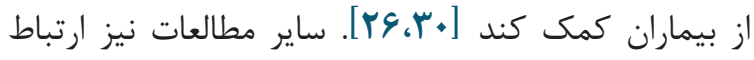

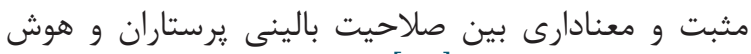

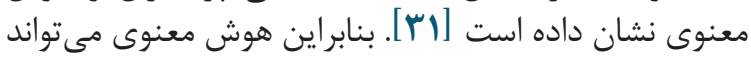

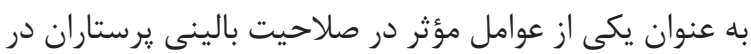

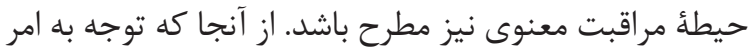

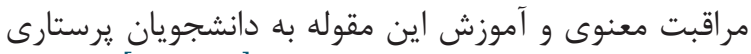

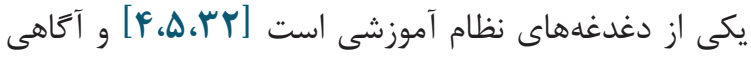

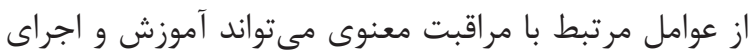

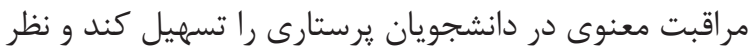

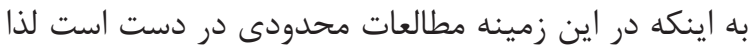

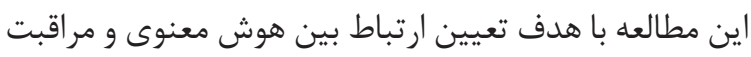
معنوى در دانشجويان يرستارى انجام شده است مئ مين

\section{روش كار}

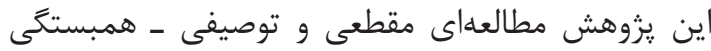

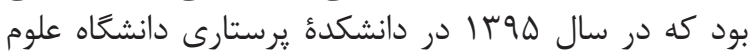

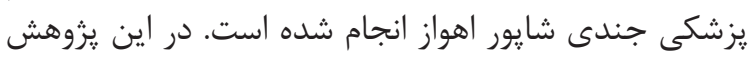

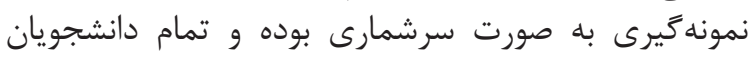

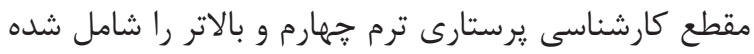

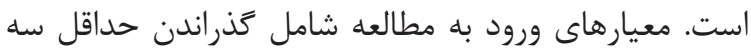

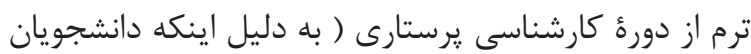

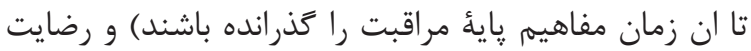

براى شركت در يزوهش بودي

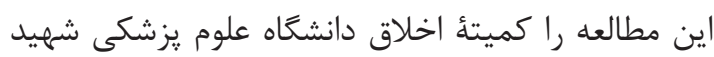
بهشتى تصويب كرد (كد اخلاق: . IR.SBMU.RETECH. مراجع ذي (1).REC

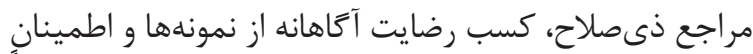

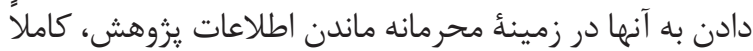

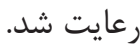

ابزار جمعآورى اطلاعات در اين مطالعه شامل يرسشنامئ

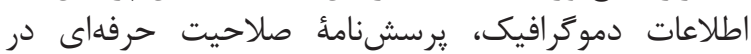

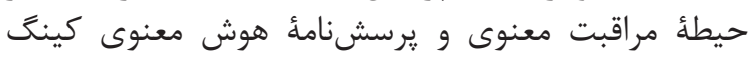

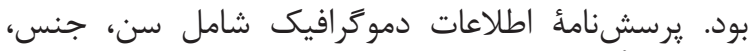

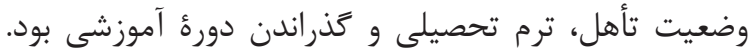

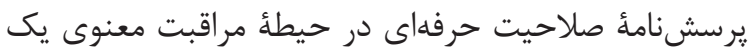

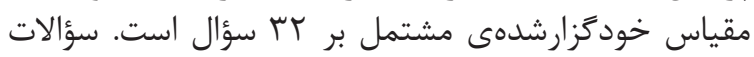

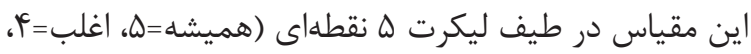

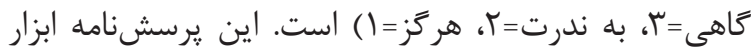

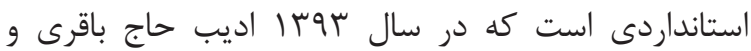

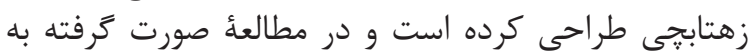

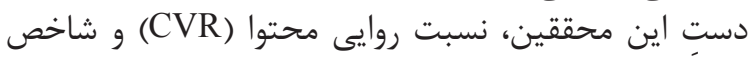

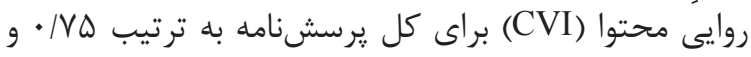

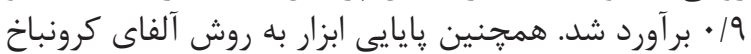

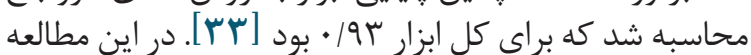




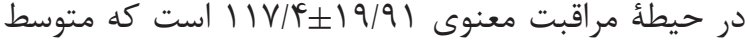

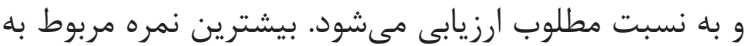

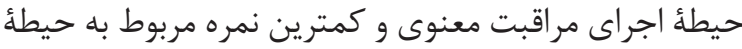
خودشناسى بود (جدول (1).

ميانحين هوش معنوى دانشجويان نيز

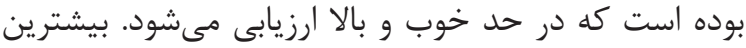

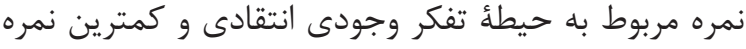

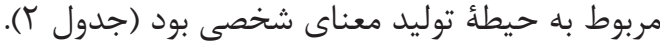

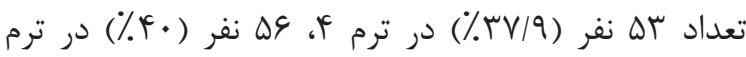

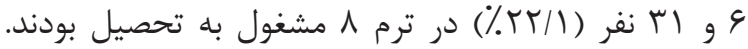
ميانگين سنى دانشجويان

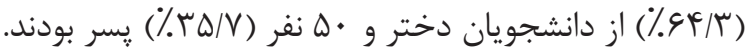

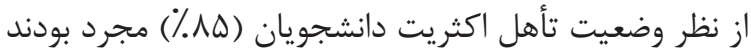

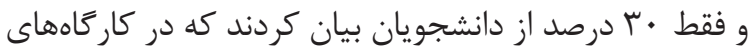

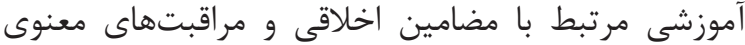
شركت كردهاند.

نتايج نشان داد كه ميانخين صلاحيت حرفهاى دانشجويان

جدول شماره ا. ميانغَين و انحراف معيار صلاحيت حرفهاى در حيطهُ مراقبت معنوى در دانشجويان يرستارى

\begin{tabular}{|c|c|}
\hline ميانتين و انحراف معيار & حيطه هاى مراقبت معنوى \\
\hline$\Delta Q / 9 T \pm I r / \Delta V$ & اجراى مراقبت معنوى \\
\hline$r r / q 9 \pm r / q \uparrow$ & ارزش هاى انسانى \\
\hline $\mid F / \cdot r \pm r / r$ & آكاهى \\
\hline $11 / G V \pm T / T \varphi$ & 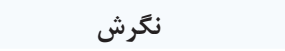 \\
\hline$N / f \pm 1 / \uparrow \varepsilon$ & خودشناسى \\
\hline$|1 V / 4 \pm| 9 / 9 \mid$ & نمرءٔ كل مراقبت معنوى \\
\hline
\end{tabular}

جدول شمارة r. ميانغين و انحراف معيار هوش معنوى دانشجويان ثبرستارى

\begin{tabular}{|c|c|}
\hline ميانَين و انحراف معيار & حيطه هاى هوش معنوى \\
\hline$r \mid / f I \pm r / V r$ & تفكر وجودى انتقادى \\
\hline$|\psi / \xi 9 \pm r /| r$ & توليد معناى شخصى \\
\hline$r \cdot / r T \pm r / V \Delta$ & آكاهى متعالى \\
\hline $\mid \Delta / 1 \pm \Gamma / \cdot \varphi$ & كسترش خودآكاهى \\
\hline$V \backslash / \kappa_{ \pm} \mid r / I I$ & نمرء كل هوش معنوى \\
\hline
\end{tabular}

اختلاف معنادارى بين گروههاى سنى، جنس زن زن و و مرد،

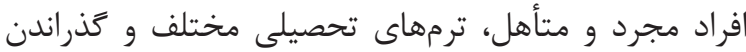

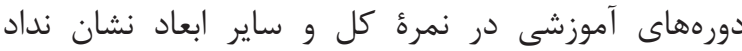
(جدول

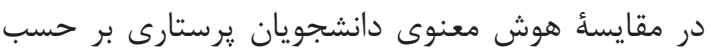

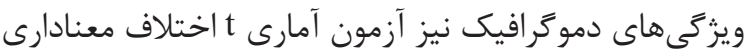

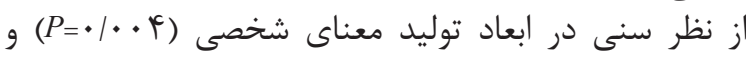

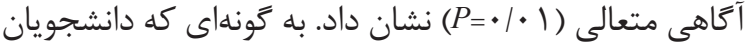
در گروه سنى بالاتر نمرههاى بيشترى كسب كردي اهن

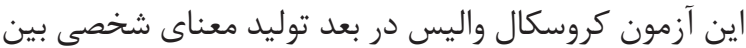

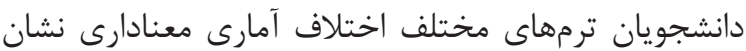

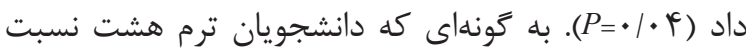

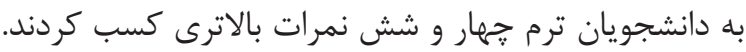

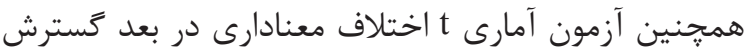

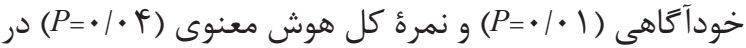

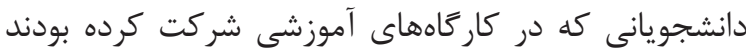

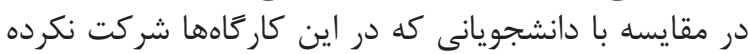
بودند نشان داد. در ساير موارد اختلاف معنادارى مانى مشاهده

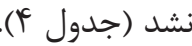

نتايج مربوط به سطح بندى نمرات كل كسب شده

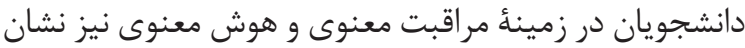

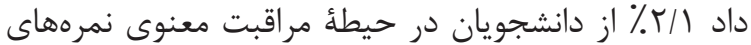

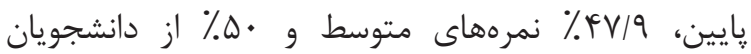

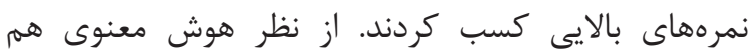

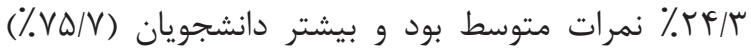
نمرهاى بالايى كسب كردند.

در مقايسأ صلاحيت حرفهاى دانشجويان :رستارى در

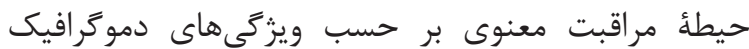

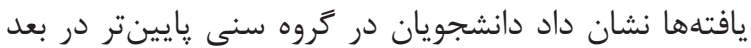

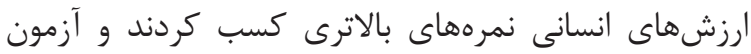

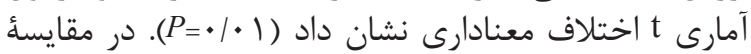

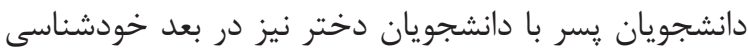

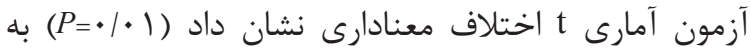

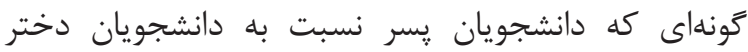

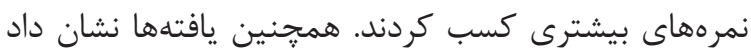

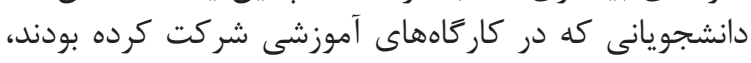

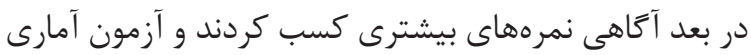

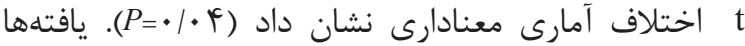




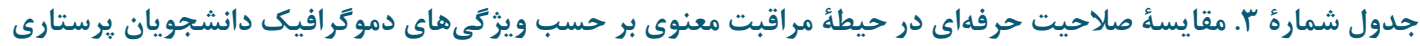

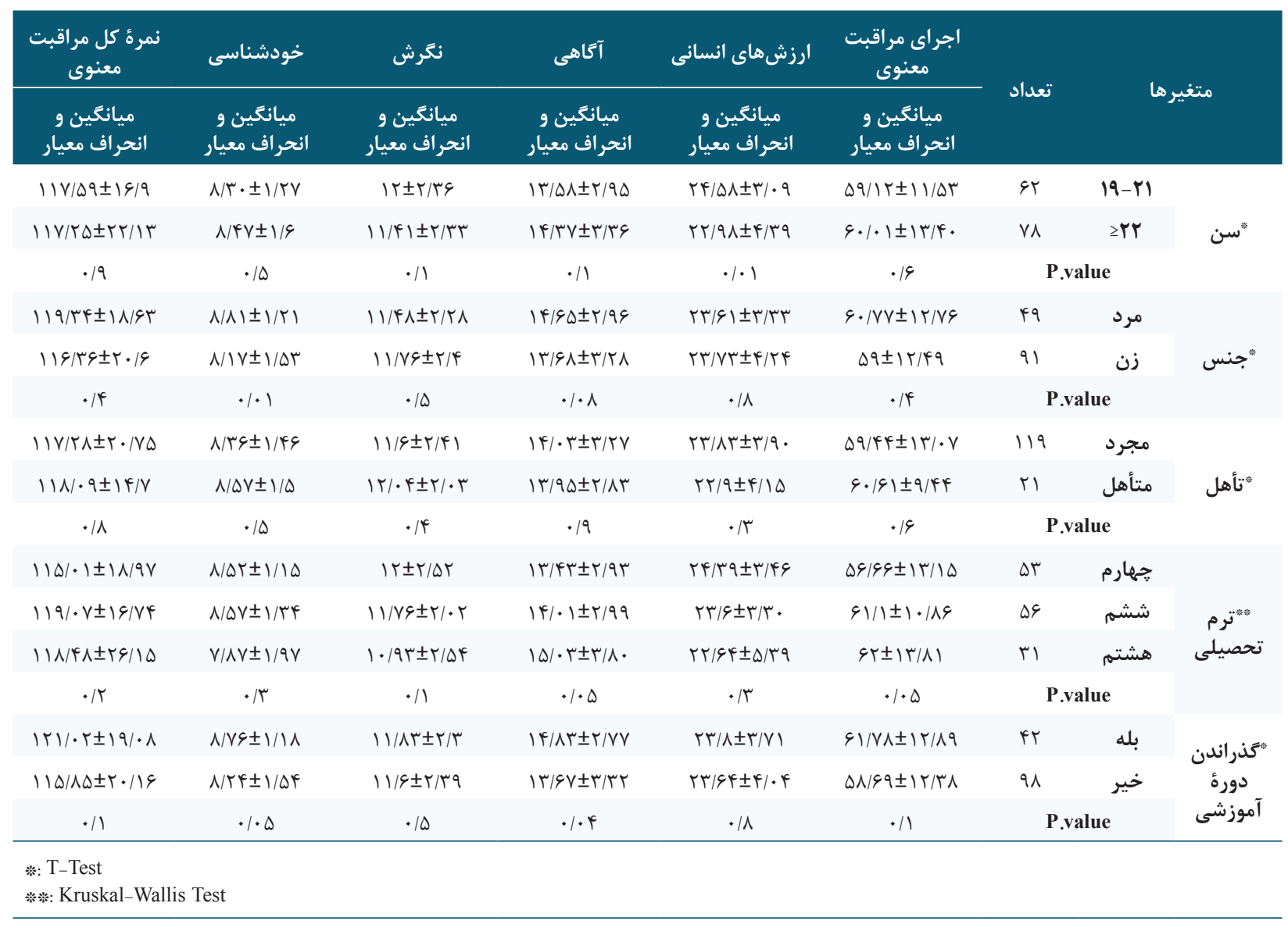

جدول شماره F. مقايسُٔ هوش معنوى بر حسب ويثزى هاى دموكر افيك دانشجويان يرستارى

\begin{tabular}{|c|c|c|c|c|c|c|c|}
\hline نمرة كل هوش & تَّترش خود & آكاهى متعالى & شخصيد معناى & تفكر انتقادى & \multirow{2}{*}{ ت تعداد } & \multirow{2}{*}{\multicolumn{2}{|c|}{ متغير ها }} \\
\hline انحراف معيار & انحر افيانَين معيار & انحراف معيار & انحراف معيار و & انحراف معيار & & & \\
\hline$q 9 / V \varphi \pm 1 \cdot / r q$ & $\mid \Psi / \Gamma \Lambda \pm r / \Delta \Lambda$ & $r \cdot / \& \Delta \pm T / \Delta r$ & $\mid r / \Lambda r \pm r / \Lambda V$ & $r 1 / \cdot 9 \pm r / 1$ & Gr & $|9-r|$ & \multirow[b]{5}{*}{ "جنس":" } \\
\hline$V Y / V \Lambda \pm|r / T|$ & $\mid Q / \varnothing 9 \pm \Psi / r$. & $r \cdot / \cdot \Delta \pm r / q r$ & $\mid \Delta / \Gamma V \pm \Psi / \backslash \Lambda$ & $r \mid / 9 q \pm r / 19$ & vı & $2 r r$ & \\
\hline$\cdot / 1$ & $\cdot 1 \cdot 1$ & $\cdot 10$ & 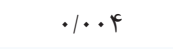 & $\cdot / r$ & \multicolumn{2}{|c|}{ P.value } & \\
\hline$V T / r T \pm 11 / \cdot \Delta$ & $\mid \nvdash / \wedge q \pm r / q \uparrow$ & 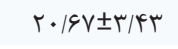 & $\mid \Delta / 1 \Lambda \pm r / q F$ & $r / / \Delta V \pm r / F$ & pq & مرد & \\
\hline$V \cdot / 9 \Delta \pm 1 r / 9 V$ & $|\Delta / r \cdot \pm r /| F$ & $\mid 9 / 9 \wedge \pm r / 91$ & $|F / F T \pm T / T|$ & $r \mid / r T \pm r / q 1$ & 91 & 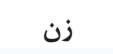 & \\
\hline$\cdot / 0$ & $\cdot 10$ & $\cdot \pi$ & $\cdot 11$ & $\cdot / V$ & \multicolumn{2}{|c|}{ P.value } & \\
\hline$V I / \cdot \Delta \pm I T / \mu r$ & $\mid \varphi / q \varphi \pm \psi / \cdot \varphi$ & $r \cdot / \Lambda \pm \pm r / v \varepsilon$ & $|F / g r \pm r /| \Lambda$ & $r \mid r \pm r / V r$ & 119 & مجرد & \multirow{3}{*}{ 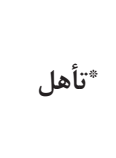 } \\
\hline$V \Psi / \Delta V \pm 1 \cdot / \wedge \Delta$ & $\mid \Delta / 9 \Delta \pm r / \cdot r$ & $r \cdot / F V \pm r / V r$ & $\mid \Delta / \cdot १ \pm r / \wedge \varphi$ & $r r / \cdot r \pm r / V r$ & rI & متأهل & \\
\hline$\cdot / r$ & $\cdot / 1$ & $\cdot / V$ & $\cdot / \Delta$ & $\cdot / 4$ & \multicolumn{2}{|c|}{ P.value } & \\
\hline$V r / \cdot q \pm q / \Delta r$ & $|r| \Lambda| \pm r / V|$ & $r \mid / イ \pm \Psi / I$ & $\mid r / \Delta \Lambda \pm r / V r$ & $r|/ 4| \pm r / 11$ & $\Delta r$ & جهارم & \multirow{4}{*}{ تحصيلى } \\
\hline 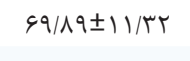 & $\mid Q / / \Psi \pm r / \Lambda \Lambda$ & $19 / \Gamma V \pm r / V \Delta$ & $\mid \psi / \kappa \varepsilon \pm \Psi / \cdot r$ & 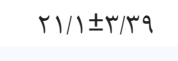 & $\Delta \varphi$ & ششم & \\
\hline$V \Psi / \cdot 9 \pm|\varepsilon / V|$ & $|Q / \Delta| \pm r / 9 \mid$ & 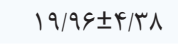 & $1 Q / G Y \pm T / \Lambda T$ & $r \mid / ৭ 9 \pm \Delta / l$ & r & هشتم & \\
\hline$\cdot / 1$ & $\cdot \pi$ & $\cdot 1 \cdot \Delta$ & $\cdot 1 \cdot 4$ & $\cdot / r$ & \multicolumn{2}{|c|}{ P.value } & \\
\hline$V F / Q T \pm \mid 1 / 91$ & $\mid 9 / \cdot r \pm r / 9 \Delta$ & $r \cdot / 9 \Delta \pm r / \Delta \varphi$ & $\mid \Delta / r \Delta \pm \Psi / 1 \Lambda$ & $r r / 19 \pm r / 9 q$ & kt & بله & \multirow{3}{*}{ "َّذرأندن } \\
\hline$V \cdot / 11 \pm 1 r / \cdot 1$ & $\mid F / V \pm r / \cdot F$ & $19 / 91 \pm \pi / \Lambda$ & $\mid f / F \pm r / \cdot \Lambda$ & $r \mid / \cdot \wedge \pm r / V I$ & 91 & خير & \\
\hline .1 .4 & $.1 \cdot 1$ & .11 & .11 &.$/ 1$ & \multicolumn{2}{|c|}{ P.value } & \\
\hline
\end{tabular}

*: T-Test

***: Kruskal-Wallis Test 
ضريب همبستخى متغير هوش معنوى با مراقبت معنوى سץ/• بوده است و اين متغير توانسته است،

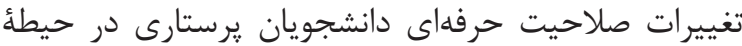

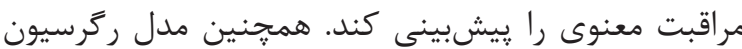

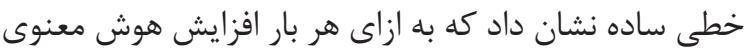

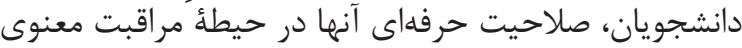

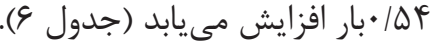

ضريب همبستخَى ييرسون ارتباط مثبت و معنادارى بين

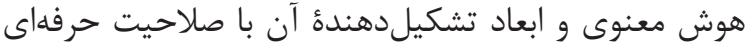

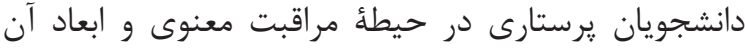

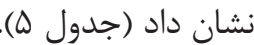

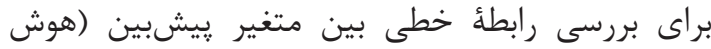

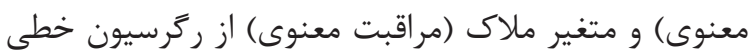

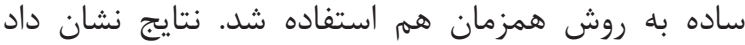

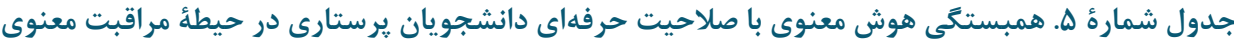

\begin{tabular}{|c|c|c|c|c|c|}
\hline نمرة كل هوش معنوى & تَسترش خود آكاهى & آكاهى متعالى & توليد معناى شخصى & تفكر انتقادى وجودى & is \\
\hline $\mathbf{r}$ (p value) & $\mathbf{r}$ (p value) & $\mathbf{r}$ (p value) & $\mathbf{r}$ (p value) & $\mathbf{r}$ (p value) & "يطظه \\
\hline$\cdot / r \varepsilon(\cdot / \cdot r)$ & $\cdot / r(\cdot / \cdot 1)$ & $\cdot / r \mid(\cdot / \cdot 1)$ & $\cdot / r(\cdot / \cdot 1)$ & $\cdot \pi(<\cdot / \cdot \cdot 1)$ & اجراى مراقبت معنوى \\
\hline$\cdot \mid \pi r(\cdot / \cdot \Delta)$ & $\cdot / r(\cdot / \cdot 1)$ & $\cdot / r F(\cdot / \cdot r)$ &.$/ 19(\cdot / \cdot r)$ & $\cdot \pi r(\cdot / \cdot \cdot \varphi)$ & ارزشهاى انسانى \\
\hline$\cdot \pi v(<\cdot / \cdot \cdot 1)$ & $\cdot / r q(\cdot / \cdot r)$ & ( & $\cdot \pi \Delta(<\cdot / \cdot \cdot 1)$ & $\cdot \pi v(<\cdot / \cdot \cdot 1)$ & آكاهى \\
\hline$\cdot / r V(\cdot / \cdot \cdot 1)$ & $\cdot / r(\cdot / \cdot 1)$ & $\cdot / r \Lambda(\cdot / \cdot \cdot 1)$ & $\cdot / 19(\cdot / \cdot r)$ & $\cdot / K \varepsilon(\cdot / \cdot .1)$ & نعَرش \\
\hline$\cdot / r \Delta(<\cdot / \cdot \cdot 1)$ & $\cdot / \pi(<\cdot / \cdot \cdot 1)$ & • & $\cdot / T V(\cdot / \cdot \cdot 1)$ & • & خودشناسى \\
\hline$\cdot \pi r(<\cdot / \cdot \cdot 1)$ & $\cdot \pi F(\cdot / \cdot r)$ & $\cdot / r q(<\cdot / \cdot \cdot 1)$ & $\cdot / \pi \&(\cdot / . .1)$ & $\cdot / r \Delta(<\cdot / \cdot \cdot 1)$ & نمرءٔ كل مراقبت معنوى \\
\hline
\end{tabular}

جدول شمارة 9 ـ مدل ركر سيونى هوش معنوى بر مراقبت معنوى

\begin{tabular}{|c|c|c|c|c|c|c|c|c|c|c|c|}
\hline p & $\mathbf{t}$ & $\beta$ & SE & B & DfY & Df) & $\mathbf{F}$ & Adj. $\mathbf{R}^{r}$ & $\mathbf{R}^{r}$ & $\mathbf{R}$ & متغير بيش بين \\
\hline$<\cdot / \cdot \cdot 1$ & - & - & - & - & IrN & 1 & $I V / V$ & $\cdot 11$. & .111 & سא/. & مدل مدل \\
\hline$<\cdot|\cdot \cdot|$ & $1 / 199$ & - & $9 / \Delta 9 \Lambda$ & VA/FYI & - & - & - & - & - & - & Constant \\
\hline$<\cdot|\cdot \cdot|$ & r/l & אTr/. & TH & $\cdot|\Delta| F C$ & - & - & - & - & - & - & هوش معنوى \\
\hline
\end{tabular}

حفظ كردند و همانند يرستاران شاغل در بخش هار با با مسائلى في

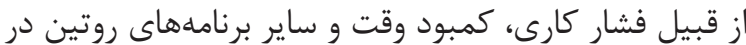

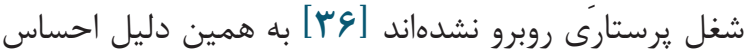

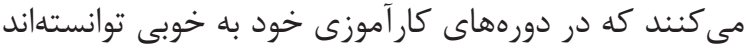

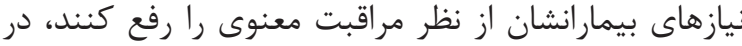
نتيجه نمرات بالايى در اين زمينه كسب كران كردند. نئن

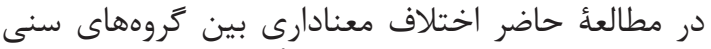

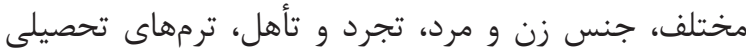

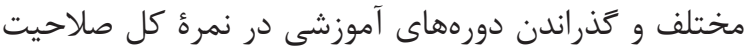

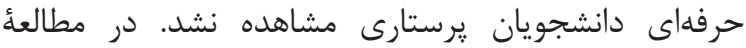

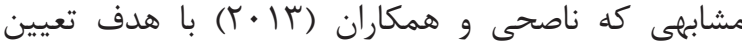

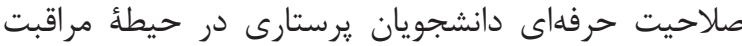

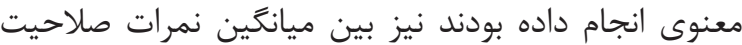

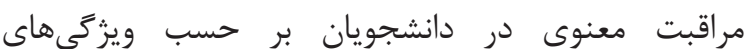

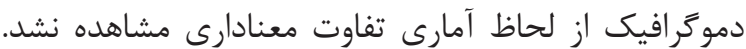

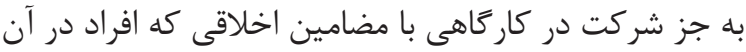

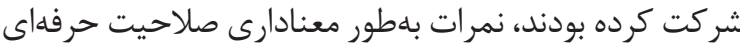

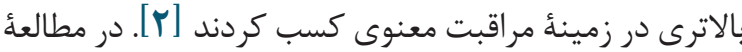

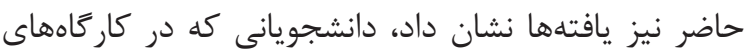

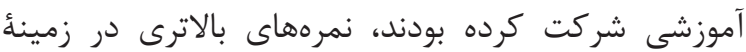

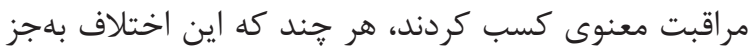

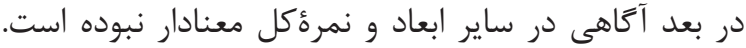

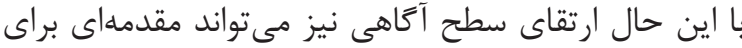
بالا رفتن صلاحيت حرفهاى دانشجويان باشي آند كه حائز اهميت

هدف اين مطالعه تعيين صلاحيت حرفهاى دانشجويان

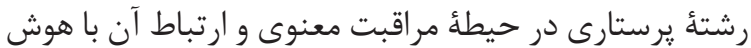

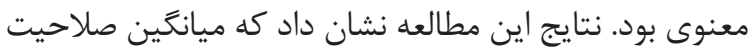

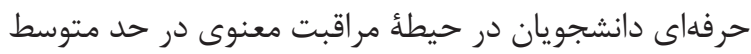

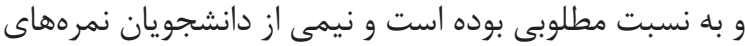

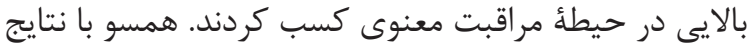

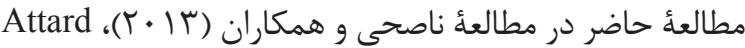

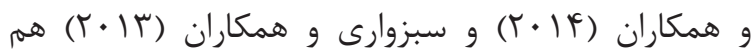
صلاحيت حرفهاى دانشجويان زيرستارى در حيطة مرأ مراقبت

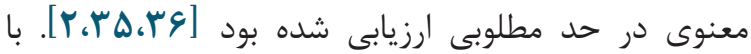

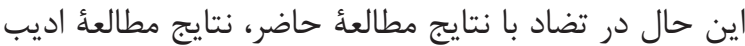

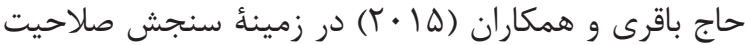

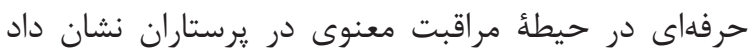

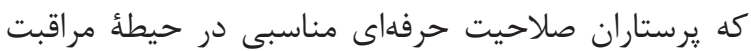

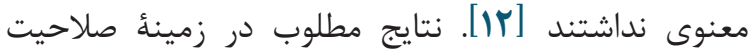

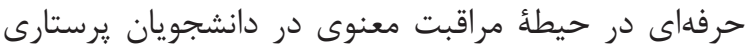

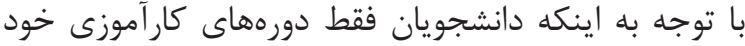

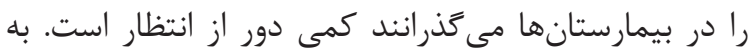

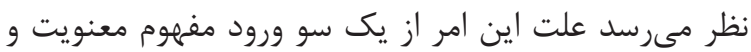

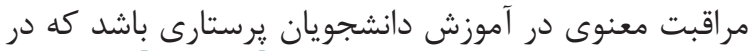

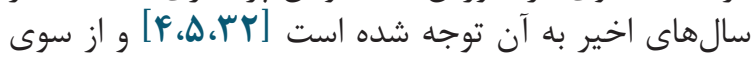

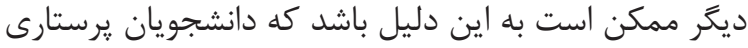

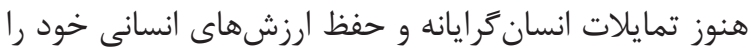


معنوى با افزايش سن باشد [ج"]

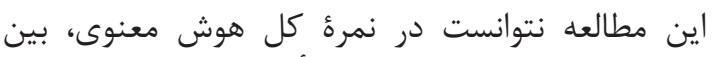

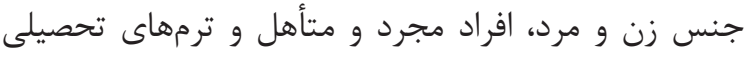

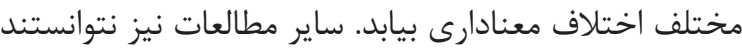

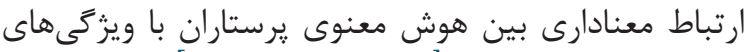

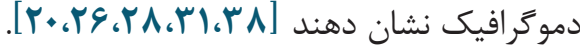

نتايج مطالعهُ حاضر بين هوش معنوى و ابعاد تشكيل دهندة

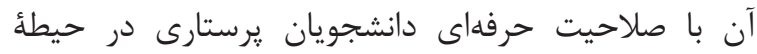

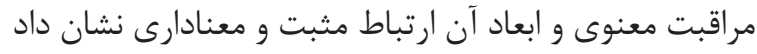

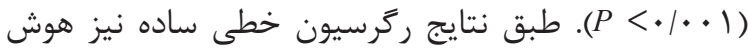

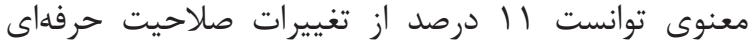

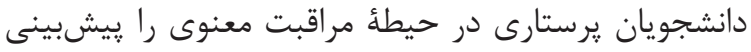

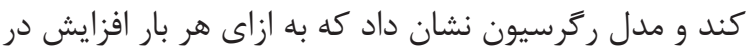

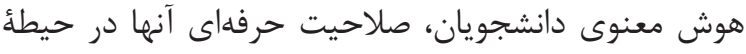

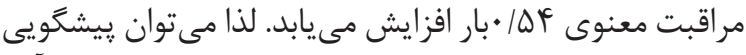

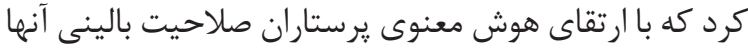

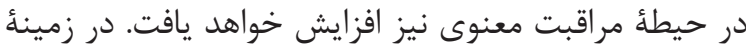

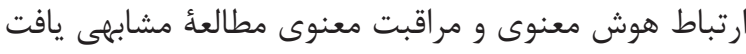

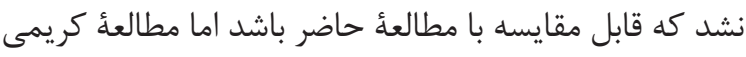

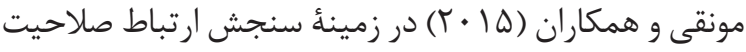

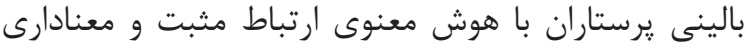

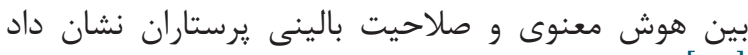

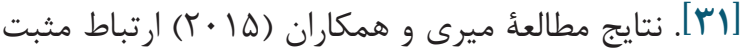

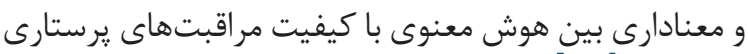

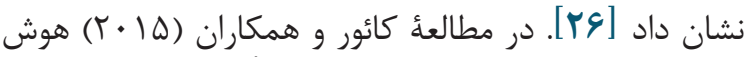

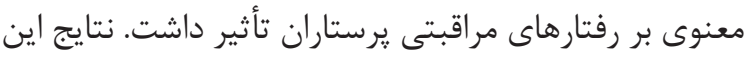

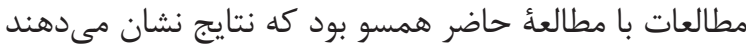

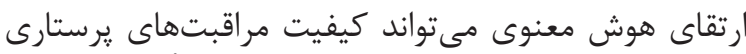

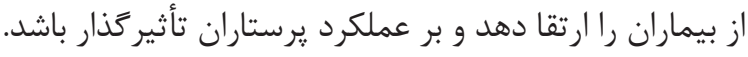

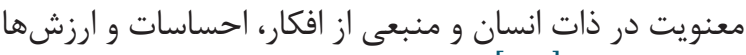

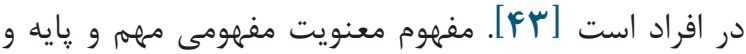

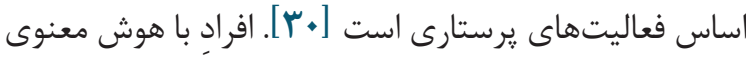

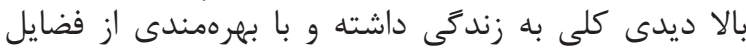

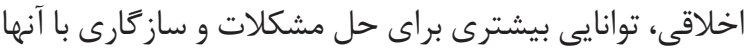

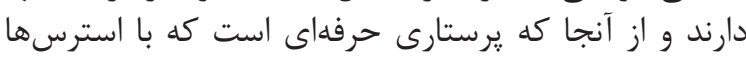

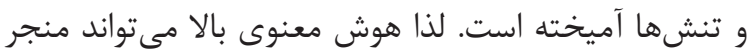

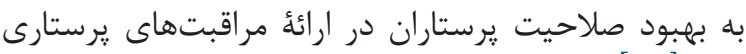

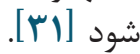

\section{نتيجه}

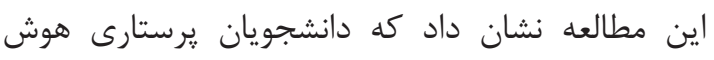

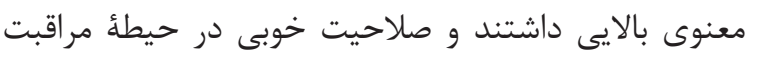

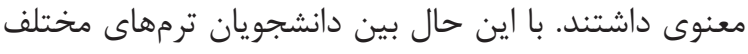

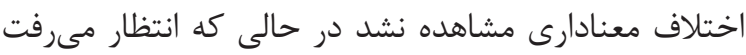

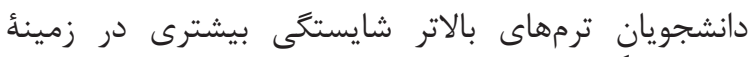

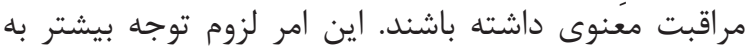

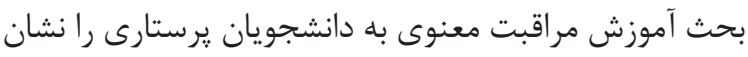

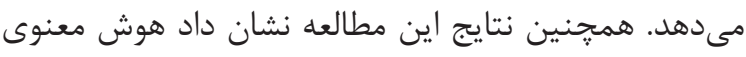

است و از اين نظر تا حدودى نتايج مطالعهُ ناصحى با مطالعه

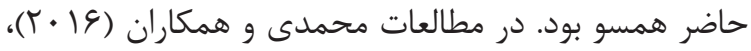

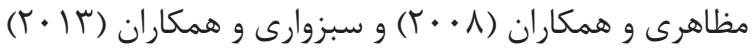

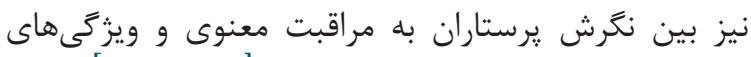

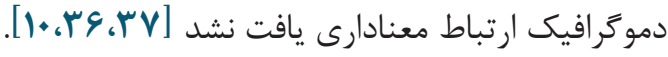

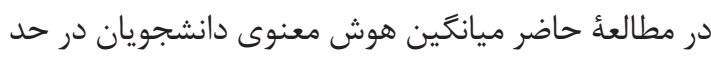

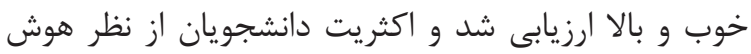

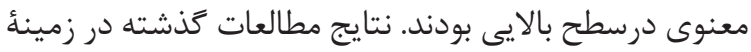

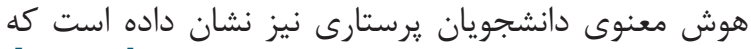

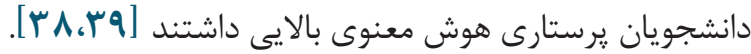

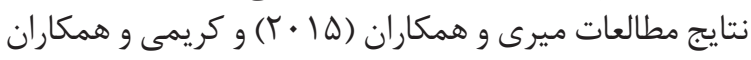

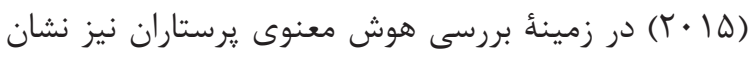

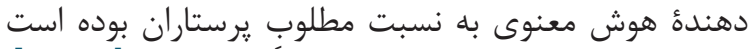

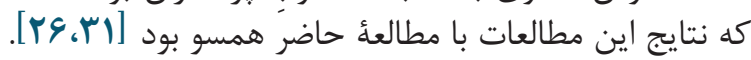

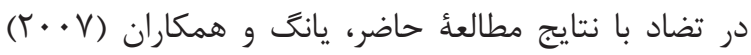

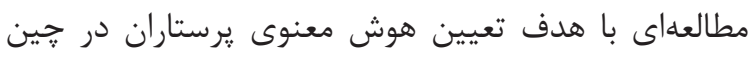

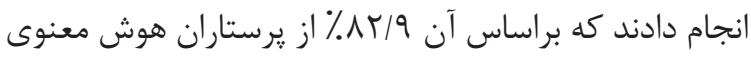

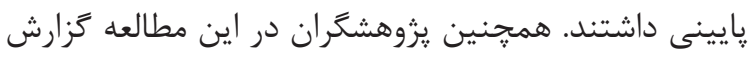

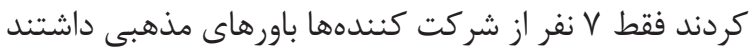

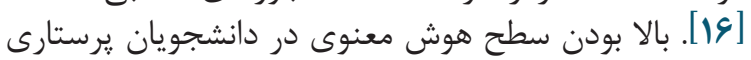

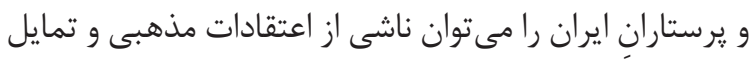

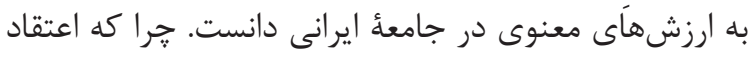

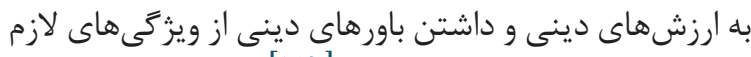

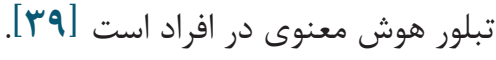

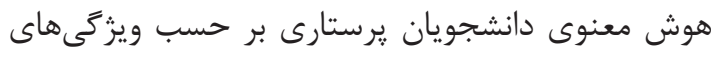

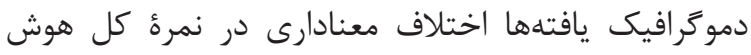

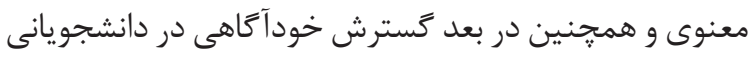

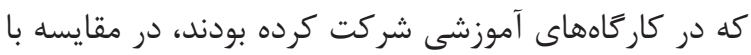

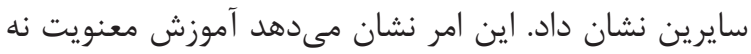

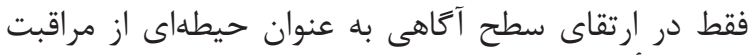

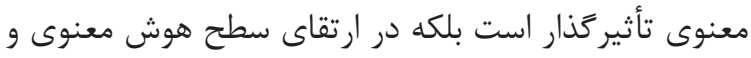

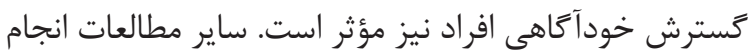

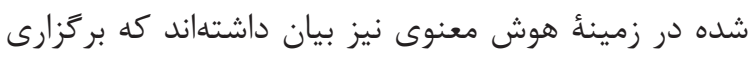

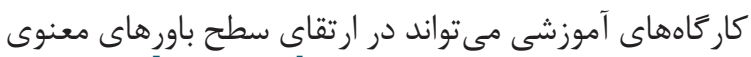

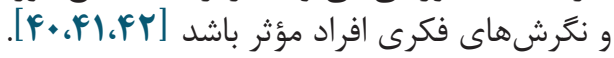

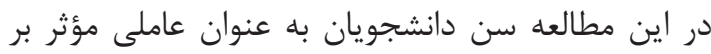

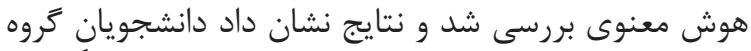

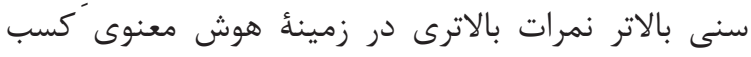

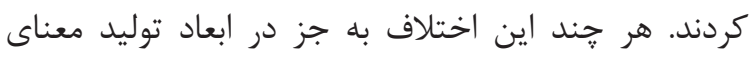

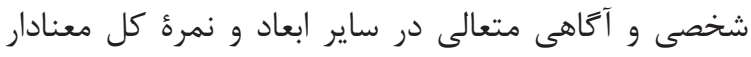

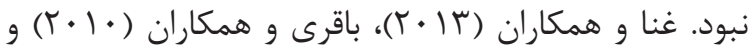

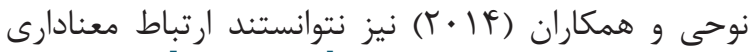

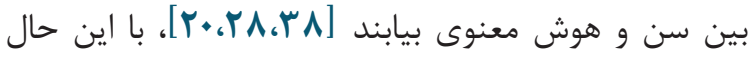

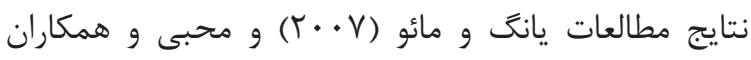

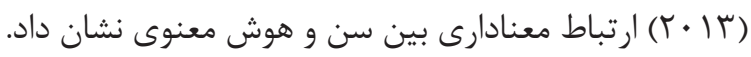

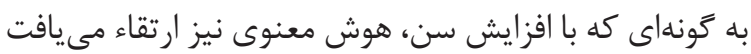

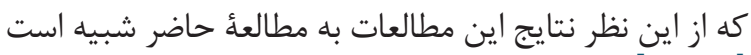

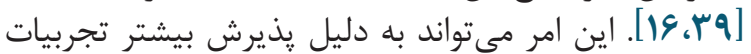




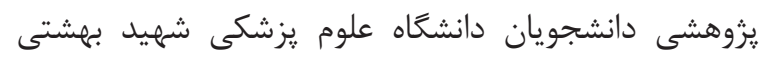

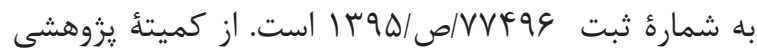

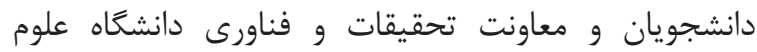
يزشكى شهيد بهشتى بران براى حمايت مالى إنى ائن اين مطالعه

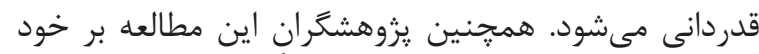

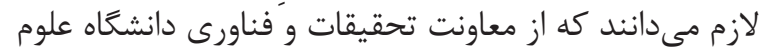

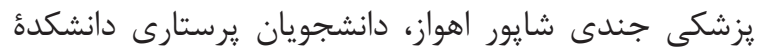

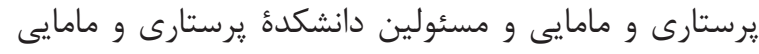

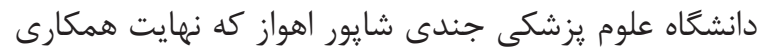

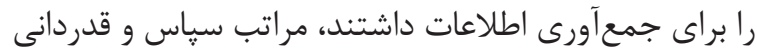

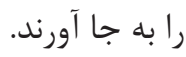

$$
\text { تعارض در منافع }
$$

بين نويسند أن در اين مطالعه تضاد منافعى وجود ندارد.

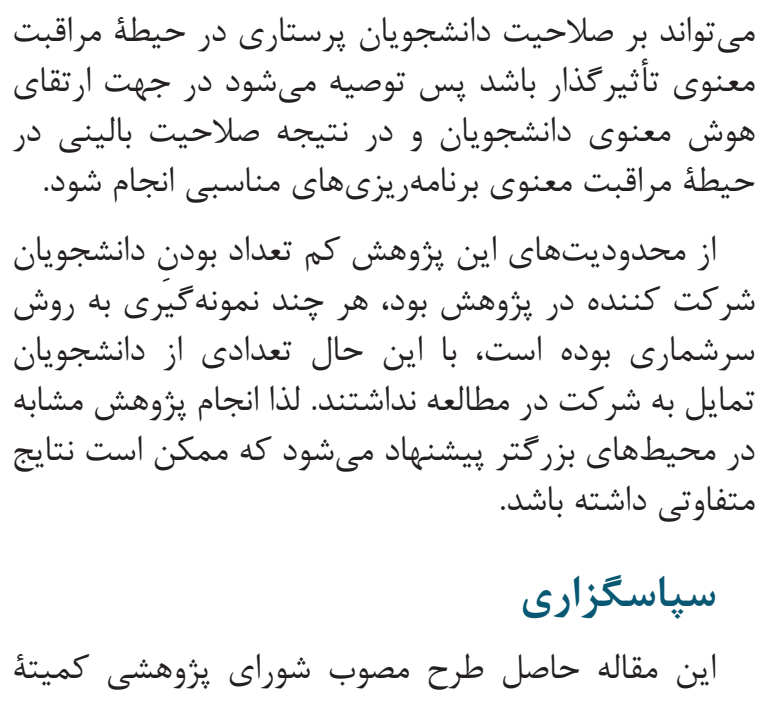

\section{References}

1. Ross L, van Leeuwen R, Baldacchino D, Giske T, McSherry W, Narayanasamy A, et al. Student nurses perceptions of spirituality and competence in delivering spiritual care: a European pilot study. Nurse Educ Today. 2014;34(5):697702. https://doi.org/10.1016/j.nedt.2013.09.014 PMid:24119953

2. Nasehi A, Rafiei H, Jafari M, Borhani F, Sabzevari S, Baneshi MR, Rahimi-Madiseh M. Survey of nurse's students competencies for delivering spiritual care to their patients. Journal of Clinical Nursing and Midwifery. 2013;4(3):1-9.

3. Zare A, Jahandideh S. The impact of special wards nursing spiritual well-being upon patients' spiritual care. Iran J Nurs Res. 2014;9(3):30-8.

4. Bennett V, Thompson ML. Teaching spirituality to student nurses. J Nurs Educ Pract. 2015;5(2):26-33.

5. Giske T. How undergraduate nursing students learn to care for patients spiritually in clinical studies--a review of literature. J Nurs Manag. 2012;20(8):1049-57. https://doi.org/10.1111/ jonm.12019

6. Baldacchino DR. Spiritual care education of health care professionals. Religions. 2015;6(2):594-613. $\quad$ https://doi.org/10.3390/ rel6020594

7. Wong KF, Yau SY. Nurses' experiences in spirituality and spiritual care in Hong Kong. Appl Nurs Res. 2010;23(4):242-44. https://doi. org/10.1016/j.apnr.2008.10.002 PMid:21035036

8. Lind B, Sendelbach S, Steen S. Effects of a spirituality training program for nurses on patients in a progressive care unit. Critical Care Nurse. 2011;31(3):87-90. https://doi.org/10.4037/ $\underline{\text { ccn2011372 PMid:21632597 }}$

9. Baldacchino DR. Teaching on the spiritual dimension in care to undergraduate nursing students: The content and teaching methods. Nurse
Education Today. 2008;28(5):550-62. https://doi. org/10.1016/j.nedt.2007.09.003 PMid: 17950959

10. Mohammadi G, Mirshahi F, Movahedi A, Taghavi M, Pasha AM, Mardani M, Kavosi A. Nurses and midwives attitude of hospitals of Neyshabur city about spirituality and spiritual care. Medical Ethics. 2016;9(33):45-63.

11. Wong K. Lee L. Lee J. Hong Kong enrolled nurses' perceptions of spirituality and spiritual care. Int Nurs Rev. 2008;55(3):333-40. https:// doi.org/10.1111/j.1466-7657.2008.00619.x PMid:19522951

12. Adib-Hajbaghery M, Zehtabchi S. Assessment of nurses' professional competence in spiritual care in Kashan's hospitals in 2014. Sci J Hamdan Univ Med Sci. 2014;22(4):23-32.

13. Chan MF, Chung LYF, Lee ASC, Wong WK, Lee GSC, Lau CY, et al. Investigating spiritual care perceptions and practice patterns in Hong Kong nurses: Results of a cluster analysis. Nurse Education Today. 2006;26(2):139-50. https://doi. org/10.1016/j.nedt.2005.08.006 PMid:16213063

14. Çetinkaya B, Azak A, Dündar S. Nurses' perceptions of spirituality and spiritual care. Aust J Adv Nurs. 2013;31(1):5-10.

15. Lewinson LP, McSherry W, Kevern P. Spirituality in pre-registration nurse education and practice: a review of the literature. Nurse Educ Today. 2015;35(6):806-14. https://doi.org/10.1016/j. nedt.2015.01.011

16. Yang K, Mao X. A study of nurses spiritual intelligence: A cross-sectional questionnaire survey. Int J Nurs Stud. 2007;44(6):999-1010. https:// doi.org/10.1016/j.ijnurstu.2006.03.004

17. Narayanasamy A, Owens J. A critical incident study of nurses' responses to the spiritual needs of their patients. Journal of Advanced Nursing. 2001;33(4):446-55. $\quad$ https://doi.org/10.1046/ j.1365-2648.2001.01690.x PMid: 11251732 


$$
\text { 191. ارتباط بين صلاحيت حرفهاى..... }
$$

18. Benjamins MR. Religion and functional health among the elderly: is there a relationship and is it constant? J Aging Health. 2004;16(3):35574. https://doi.org/10.1177/0898264304264204 PMid:15155067

19. Speck P, Higginson I, Addington-Hall J. Spiritual needs in health care. BMJ. 2004;329(7458):1234. $\quad$ https://doi.org/10.1136/bmj.329.7458.123 PMid: 15258045

20. Ghana S, Jouybari LM, Sharifnia SH, Hekmat Afshar M, Sanagoo A, Chehreh Gosha M. Correlation of spiritual intelligence with some of demographic and educational factors among the students of Golestan University of Medical Sciences. Journal of Health Promotion. 2013;2(1):17-23.

21. Shiasi M, Etebarian A, Zarin Abadi Z, Ahmadi M. The effect of spiritual intelligence on the productivity of employees at Isfahan University of Medical Sciences based on structural equation model. Journal of Health Administration. 2016;19(63):34-44.

22. King DB. Rethinking claims of spiritual intelligence: A definition, model, and measure. Bethesda, MD: ProQuest; 2008.

23. Noble, K. D. Spiritual intelligence: A new frame of mind. Advanced Development Journal. 2000;9:1-29.

24. Nouhi E, Nakhaee N, Rahimi N. Spiritual intelligence and attitude towards spirituality and spiritual care in nursing and midwifery students. Iran J Nurs Res. 2014;27(90):150-9.

25. Mashayekhi Dolatabadi MR, Mohammadi M. Resilience and spiritual intelligence predictors of as academic self-efficacy in urban and rural students. Journal of school psychology. 2014;3(2):205-25.

26. Miri KH, Keshavarz A, Shirdelzadeh S, Parsa P. The relationship between nurses' spiritual Intelligence and quality of nursing care based on Nurses' \& patients' viewpoints. J Urmia Nurs Midwifery Fac. 2015;13(6):518-24.

27. Amram Y. The contribution of emotional and spiritual intelligences to effective business leadership.[disertation]. Palo Alto: Institute of Transpersonal Psychology; 2009.

28. Bagheri F, Akbarizadeh F, Hatami H. The relationship between nurses' spiritual intelligence and happiness in Iran. Procedia Soc Behav Sci. 2010;5:1556-61. https://doi.org/10.1016/j.sbspro.2010.07.325

29. Ghaleei A, Mohajeran B, Mahmoodzadeh M. The relationship among spiritual intelligence, mental health and job stress in nurses in imam khomeini hospital of mahabad. Sci J Hamdan Univ Med Sci. 2016;23(4):14-21

30. Kaur D, Sambasivan M, Kumar N. Impact of emotional intelligence and spiritual intelligence on the caring behavior of nurses: a dimension-level exploratory study among public hospitals in Malaysia. Appl Nurs Res. 2015;28(4):2938. https://doi.org/10.1016/j.apnr.2015.01.006 PMid:26608428

31. Karimi-Moonaghi H, Gazerani A, Vaghee S, Gholami H, Salehmoghaddam AR, Gharibnavaz $\mathrm{R}$. Relation between spiritual intelligence and clinical competency of nurses in Iran. Iran J Nurs Midwifery Res. 2015;20(6):665-9. https://doi. org/10.4103/1735-9066.170002 PMid:26793250

32. Ali G, Wattis J, Snowden M. 'Why are spiritual aspects of care so hard to address in nursing education?' a literature review (1993-2015). International Journal of Multidisciplinary Comparative Studies. 2015;2(1):7-31.

33. AdibHajbaghery M, Zehtabchi S. Developing and validating an instrument to assess the nurses' professional competence in spiritual care. Journal of Nursing Measurement. 2016;24(1):1527. https://doi.org/10.1891/1061-3749.24.1.15 PMid:27103239

34. Hosein Chari M, Zakeri HM. The effect of university majors, religious and artistic sciences on the spiritual intelligence; an attempt to validation and reliability measurement of the scale of spiritual intelligence. Period Edu Measur. 2010;1(1):71-92.

35. Attard J, Baldacchino DR, Camiller L. Nurses' and midwives' acquisition of competency in spiritual care: A focus on education. Nurse Education Today. 2014;1(1):1-7. https://doi.org/10.1016/j. nedt.2014.04.015

36. Sabsevari S, Jafari M, Bonashi M, Borhani F. Competence spiritual care in nursing and nursing students of Kerman University of Medical Sciences. Journal of Medical Jurisprudence. 2013;5(15,16):123-38.

37. Mazaheri M, Falahi Khoshknab M, Madah B, Rahgozar M. Attitude of nursing to spirituality and spiritual care. Payesh Journal. 2009;8(1):317.

38. Noohi E, Rahimi N, Nakhaee N. Study spiritual intelligence nursing and midwife students of kerman university of medical sciences in 1391. Medical History Journal. 2014;5(17):63-78.

39. Mohebi P, Rastegari L, Jafari A, Sepehrinia M. Spiritual intelligence in nursing students and its relationship with some demographic variables in Zanjan school of nursing and midwifery. Nursing and Midwifery Care Journal. 2013;2(2):49-56.

40. Golchin M, Sanjari AR. The relation between spiritual intelligence and self efficacy in employees (Case study: Golestan Province Education Department). Organizational Behavior Studies Quarterly. 2013;1(3):54-66. 
41. Ghasemi-Pirbalouti M, Ahmadi R, Alavi-Eshgaftaki SM, Alavi-Eshkaftaki SS. The association of spiritual intelligence and job satisfaction with mental health among personnel in Shahrekord University of Medical Sciences. J Shahrekord Univ Med Sci. 2014;16(5):123-31.

42. Pakniat M, Javidy H. Mediating role of citizenship behaviors for spiritual intelligent and organizational mental health. Journal of psychological models and methods. 2016;7(23):19-37.

43. Hosseini M, Elias H, Krauss SE, Aishah S. A review study on spiritual intelligence, adolescence and spiritual intelligence: factors that may contribute to individual differences in spiritual intelligence and the related theories. Journal of Social Sciences. 2010;6(3):429-38. https://doi. org/10.3844/jssp.2010.429.438 